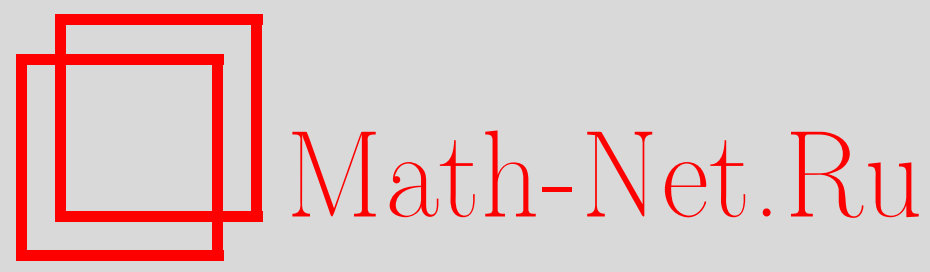

С. Ю. Доброхотов, М. А. Потеряхин, О нормальных формах в окрестности двумерных резонансных торов для многомерного ангармонического осциллятора, Матем. заметки, 2004, том 76, выпуск 5, 701-713

DOI: https://doi.org/10.4213/mzm140

Использование Общероссийского математического портала Math-Net.Ru подразумевает, что вы прочитали и согласны с пользовательским соглашением http://www.mathnet.ru/rus/agreement

Параметры загрузки:

IP: 54.197 .217 .227

26 апреля 2023 г., 15:30:47

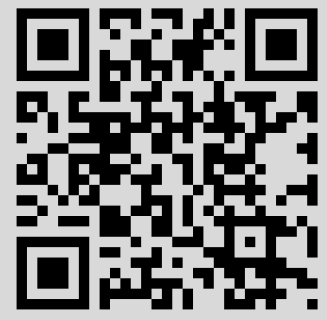




\title{
О НОРМАЛЬНЫХ ФОРМАХ \\ В ОКРЕСТНОСТИ ДВУМЕРНЫХ РЕЗОНАНСНЫХ ТОРОВ ДЛЯ МНОГОМЕРНОГО АНГАРМОНИЧЕСКОГО ОСЦИЛЛЯТОРА
}

\author{
С. Ю. Доброхотов, М.А. Потеряхин
}

В работе рассматривается задача построения нормальной формы в окрестности двумерного инвариантного изотропного тора для многомерного ангармонического осциллятора. Построена нормальная форма четвертого порядка по переменным типа гармонического осциллятора. Показано, что наличие резонансов приводит к неполиномиальной зависимости нормальной формы от переменных типа действия.

Библиографоия: 17 названий.

1. Введение. В работе рассматривается задача построения нормальной формы в окрестности инвариантных многообразий для $(n+2)$-мерного ангармонического осциллятора, задаваемого гамильтонианом вида

$$
H=\frac{P_{1}^{2}+\omega_{1}^{2} Q_{1}^{2}}{2}+\frac{P_{2}^{2}+\omega_{2}^{2} Q_{2}^{2}}{2}+\frac{1}{2}\left\langle r, \Gamma\left(Q_{1}, Q_{2}\right) r\right\rangle, \quad r={ }^{t}(q, p) \in \mathbb{R}_{q p}^{2 n},
$$

$\Gamma-(2 n \times 2 n)$-матрица с элементами, гладко зависящими от $Q_{1}, Q_{2} ;\langle\cdot, \cdot\rangle$ означает скалярное произведение, а левый верхний индекс ${ }^{t}$ - транспонирование. Частоты $\left(\omega_{1}, \omega_{2}\right)$ являются постоянными и могут быть как несоизмеримыми, так и соизмеримыми, причем второй случай представляет для нас больший интерес.

Соответствующая (1) гамильтонова система, очевидно, допускает инвариантное многообразие, задаваемое условием $r=0$. Динамика на этом многообразии задается гамильтонианом двумерного гармонического осциллятора

$$
H_{\mathrm{osc}}=\frac{P_{1}^{2}+\omega_{1}^{2} Q_{1}^{2}}{2}+\frac{P_{2}^{2}+\omega_{2}^{2} Q_{2}^{2}}{2}
$$

и хорошо известна. Полная система, отвечающая (1), как правило, является неинтегрируемой. Мы хотим изучить вопрос о поведении ее решений в окрестности указанного инвариантного многообразия $r=0$. Известно, что для реализации этой цели одним из наиболее подходящих инструментов является апшарат нормальных форм (см. [1]-[7]), который в подходящих координатах с некоторой точностью и при некоторых условиях на соответствующие частоты позволяет заменить исходный гамильтониан $H$ на более простой, часто порождающий уже интегрируемую гамильтонову систему. Траектории

Работа выполнена при поддержке фонда INTAS, грант № 00-257. 
последней приближенно описьвают динамику в окрестности соответствующего многообразия. Такой “приближенньй” гамильтониан назьвается нормальной формой.

Как правило, подходящими переменными для построения нормальных форм оказываются переменные типа действие-угол и гармонического осциллятора, а нормальная форма имеет в нерезонансном случае вид полинома по переменным действия, отвечающим за “отклонения" траекторий от исходного инвариантного многообразия. Процедура построения нормальной формы назьвается нормализацией. Важную роль при проведении нормализации играют условия типа соизмеримости или несоизмеримости частот движения на инвариантном многообразии и решений системы уравнений в вариациях в его окрестностях.

Для рассматриваемого гамильтониана динамика на инвариантном многообразии $r=0$ в случае несоизмеримых частот $\omega_{1}$ и $\omega_{2}$ задается почти периодическими траекториями, заметающими двумерные торы. Эти торы расслаивают четырехмерное фазовое пространство с координатами $\left(Q_{1}, Q_{2}, P_{1}, P_{2}\right)$.

В дальнейшем при построении нормальных форм будут использоваться последовательные преобразования переменных. Переменные действие-угол для двумерного гармонического осциллятора задаются хорошо известными формулами

$$
Q_{j}=\sqrt{\frac{2 I_{j}^{(1)}}{\omega_{j}}} \cos \varphi_{j}^{(1)}, \quad P_{j}=\sqrt{2 \omega_{j} I_{j}^{(1)}} \sin \varphi_{j}^{(1)}, \quad j=1,2,
$$

а соответствуюшие двумерные торы определяются равенствами

$$
I_{1}^{(1)}=\text { const, } \quad I_{2}^{(1)}=\text { const . }
$$

Переменные соответствующего преобразования мы будем помечать его номером в скобках. В случае несоизмеримости частот $\omega_{1}$ и $\omega_{2}$ эти торы выбираются однозначно. Поднимая их в исходное $(2 n+2)$-мерное фазовое пространство, мы получаем двумерные изотропные торы $\Lambda^{2}\left(I^{(1)}\right)$, инвариантные относительно фазового потока $g_{H}^{t}$, порожденного уже полным гамильтонианом $H$. В переменных $\left(\varphi^{(1)}, I^{(1)}, r\right)$ они задаются равенствами (4) и $r=0$. Траектории на торах $\Lambda^{2}\left(I^{(1)}\right)$ порождают систему в вариациях:

$$
\frac{d}{d t} r=\hat{J} \Gamma^{(1)} r, \quad \varphi^{(1)}=\omega t+\varphi_{0}^{(1)}, \quad \widehat{J}=\left(\begin{array}{cc}
0 & E \\
-E & 0
\end{array}\right),
$$

$\Gamma^{(1)}=\Gamma^{(1)}\left(\varphi_{1}^{(1)}, \varphi_{2}^{(1)}, I_{1}^{(1)}, I_{2}^{(2)}\right)$. Зафиксируем некоторые $I_{1}^{(1)}, I_{2}^{(2)} \geqslant 0$. В случае несоизмеримости частот коэффициенты в системе (5), очевидно, являются почти периодическими функциями $t$. Напомним, что система (5) назьвается приводимой (см. [3]-[5], [7]), если преобразованием вида $r=V\left(\varphi^{(1)}, I^{(1)}\right) \zeta$, где $V-2 \pi$-периодическая функция по углам $\varphi_{1}^{(1)}, \varphi_{2}^{(1)}$, эта система приводится к виду $\dot{\zeta}=\widehat{M} \zeta, \zeta \in \mathbb{C}^{2 n} ; \widehat{M}$ - матрица размера $2 n \times 2 n$, зависящая от $I^{(1)}$ и не зависящая от $\varphi^{(1)}$.

Если приводимая система (5) устойчива, то матрицу $\widehat{M}$ можно считать диагональной, на диагонали которой стоят чисто мнимые показатели $\Phi$ локе $i \beta$. Тогда сушествует преобразование от переменных $r={ }^{t}(Q, P)$ к “нормальным" координатам или координатам “типа гармонического осциллятора" $\zeta=(\xi, \bar{\xi})$ (черта означает комплексное сопряжение) [8]-[10]:

$$
r=V\left(\varphi_{1}^{(j)}, \varphi_{2}^{(j)}, I_{1}^{(j)}, I_{2}^{(j)}\right) \xi+\bar{V}\left(\varphi_{1}^{(j)}, \varphi_{2}^{(j)}, I_{1}^{(j)}, I_{2}^{(j)}\right) \bar{\xi}, \quad V={ }^{t}(Z, W)
$$


где $Z, W$ - гладкие матричнозначные функции $Z\left(\varphi^{(j)}, I^{(j)}\right), W\left(\varphi^{(j)}, I^{(j)}\right), 2 \pi$-периодические по углам $\left(\varphi_{1}^{(j)}, \varphi_{2}^{(j)}\right)$, и для которых выполнены следующие свойства: $Z_{l} \bar{W}_{m}-$ $W_{l} \bar{Z}_{m}=2 i \delta_{l m}, l, m=1, \ldots, n$. В этих переменных система (5) принимает вид

$$
\frac{d}{d t}\left(\begin{array}{c}
\xi \\
\bar{\xi}
\end{array}\right)=\left(\begin{array}{cc}
B & 0 \\
0 & -B
\end{array}\right)\left(\begin{array}{l}
\xi \\
\bar{\xi}
\end{array}\right), \quad B=\left(\begin{array}{cccc}
\beta_{1} & 0 & \ldots & 0 \\
0 & \beta_{2} & \ldots & 0 \\
\ldots & \ldots & \ldots & \ldots \\
0 & \ldots & 0 & \beta_{n}
\end{array}\right)
$$

$\beta_{k}$ - показатели $\Phi$ локе, $k=1, \ldots, n$, зависяшие от $I_{1}^{(1)}, I_{2}^{(1)}$.

Заметим, что переход к переменным $\varphi^{(1)}, I^{(1)}, \xi, \bar{\xi}$ в гамильтоновой системе, отвечающей гамильтониану (1), оказьвается не каноническим; каноничность будет достигнута путем введения поправок к переменным действия $I^{(1)}$ (см. раздел 2, [4], [9]).

Если система (5) приводима и устойчива, то на основе результатов [1]-[6], [9], [11] можно показать, что в окрестности изотропного тора, фиксируемого значениями $\left(I_{1}^{(1)}=I_{1}^{0}, I_{2}^{(1)}=I_{2}^{0}, r=0\right)$, при некоторых дополнительных условиях типа нерезонансности на частоты $(\omega, \beta)$ можно построить преобразования к переменным типа действие-угол и гармонического осциллятора. Пусть после $k$ замен переменньх построено преобразование $\left(\varphi^{(1)}, I^{(1)}, \xi, \bar{\xi}\right) \rightarrow\left(\varphi^{(k)}, I^{(k)}, \xi^{(k)}, \bar{\xi}^{(k)}\right)$ и $\Delta I=I^{(k)}-I^{0}$, т.е. $\Delta I$ - суммарная поправка к действию после $k$ преобразований. Тогда при некоторых условиях типа нерезонансности частот согласно [4], [9] гамильтониан (1) принимает вид

$$
H=\left\langle\omega,\left(I^{0}+\Delta I\right)\right\rangle+\langle\beta, \rho\rangle+\sum_{i+j=2, j \geqslant 1}^{N} \mathscr{H}_{i j}\left(I^{0}\right) \Delta I^{i} \rho^{j}+O\left(|\rho|^{1 / 2}(|\Delta I|+|\rho|)^{N}\right),
$$

где $\omega=\left(\omega_{1}, \omega_{2}\right), \beta=\left(\beta_{1}, \ldots, \beta_{n}\right), \rho=\xi^{(k)} \bar{\xi}^{(k)}$. Число $N$ может быть выбрано достаточно большим; при условиях типа аналитичности невязку можно сделать экспоненциально малой. При этом заметим, что вычисления конкретных выражений для $\mathscr{H}_{i j}$ могут представлять определенные трудности даже для достаточно небольших $N$. C другой стороны, с точки зрения приложений (например, для квантовых спектральных задач [9], [12]) в (8) бьвает интересно явно вычислить коэффициенты для $N=1$ и $N=2$.

В этой работе нас в основном интересует построение формул, аналогичных (8), в резонансном случае - в случае соизмеримых частот $\omega_{1}$ и $\omega_{2}: \omega_{1} / \omega_{2}=m_{1} / m_{2}, m_{1}, m_{2} \in \mathbb{Z}$. Этот случай довольно сильно отличается от нерезонансного: все траектории двумерного гармонического осциллятора являются замкнутьги кривыми - хорошо известными фигурами Лиссажу, а в подходящих переменных гамильтониан $H_{\text {osc }}$ зависит только от одного действия (считаем, что от $I_{1}^{(2)}$ ):

$$
H_{\mathrm{osc}}=\omega_{0} I_{1}^{(2)}, \quad \omega_{0}=\frac{\omega_{1}}{m_{1}}=\frac{\omega_{2}}{m_{2}} .
$$

Один из вариантов выбора таких подходящих переменных на основе уже построенных переменных $\left(\varphi^{(1)}, I^{(1)}\right)$ определяется следующими формулами:

$$
\begin{cases}I_{1}^{(2)}=m_{1} I_{1}^{(1)}+m_{2} I_{2}^{(1)}, & \varphi_{1}^{(2)}=n_{1} \varphi_{1}^{(1)}+n_{2} \varphi_{2}^{(1)} \\ I_{2}^{(2)}=n_{2} I_{1}^{(1)}-n_{1} I_{2}^{(1)}, & \varphi_{2}^{(2)}=m_{2} \varphi_{1}^{(1)}-m_{1} \varphi_{2}^{(1)}\end{cases}
$$


где $m_{1} n_{1}+m_{2} n_{2}=1, m_{1}, n_{1}, m_{2}, n_{2} \in \mathbb{Z}^{+}$(их существование гарантируется соответствуюшими утверждениями теории чисел). Выбор $\left(n_{1}, n_{2}\right)$ не является единственным и мы фиксируем его каким-либо образом. Важно подчеркнуть, что частота $\omega_{0}$ (как и частоты $\omega_{1}$ и $\left.\omega_{2}\right)$ постоянна и не зависит от переменных действия, что делает рассматриваемую задачу отличной от резонансных задач, в которых появляются траектории Пуанкаре (см. теорему 4.8 из [3]).

В резонансном случае торы (4) остаются инвариантными относительно фазового потока $g_{H}^{t}$ и фазовое пространство полной системы по-прежнему может быть расслоено на двумерные торы, но теперь выбор таких торов уже не однозначен. Это связано с тем, что инвариантные многообразия, движение на которых задается гамильтонианом $H_{\mathrm{osc}}$, могут быть “склеены” из набора одномерных замкнутых траекторий, задаваемых значениями $\left(\varphi_{2}^{(2)}, I_{1}^{(2)}, I_{2}^{(2)}\right)$. В частности, из замкнутых траекторий можно получать различные двумерные торы, задавая при фиксированном значении $I_{1}^{(2)}=I_{1}^{0}$ различные способы "склейки" наборов таких траекторий. Один из возможных способов задания тора построить функцию $F$ в $\mathbb{R}_{Q P}^{4}$, находящуюся в инволюции с гамильтонианом $H_{\mathrm{osc}}$. В переменных $\left(\varphi^{(2)}, I^{(2)}\right)$ такая функция, очевидно, не зависит от $\varphi_{1}^{(2)}$ и тогда соответствующие двумерные торы задаются равенствами $F\left(\varphi_{2}^{(2)}, I_{1}^{(2)}, I_{2}^{(2)}\right)=$ const, $I_{1}^{(2)}=$ const при условии, что последние равенства задают компактные множества. Указанная функция $F$ может зависеть и от других параметров. На построенных торах можно ввести новые переменные действие-угол $\left(\psi_{1}, \psi_{2}, \mathscr{I}_{1}, \mathscr{I}_{2}\right)$. В этих переменньх функция $F$ будет зависеть только от $\left(\mathscr{I}_{1}, \mathscr{I}_{2}\right)$.

Вернемся к системе уравнений в вариациях (5). Теперь переменные $\varphi_{2}^{(2)}, I_{1}^{(2)}, I_{2}^{(2)}$ входят в нее как параметры и, в отличие от нерезонансного случая, она является системой с периодическими коэффициентами. Поэтому для нее применима теория Флоке-Ляпунова (см., например, [10]). Условие приводимости выполнено автоматически, но теперь матрица $\widehat{M}$ уже зависит и от угла $\varphi_{2}^{(2)}$. В случае устойчивости системы в вариациях мы можем построить преобразования типа (6) и привести ее к виду (7) с показателями Флоке, зависяшими от $I^{(2)}$ и, вообще говоря, от угла $\varphi_{2}^{(2)}$.

Мы хотим построить нормальную форму для резонансного случая. Основной результат данной работы состоит в том, что в резонансном случае нормальная форма гамильтониана в подходящих переменных при вьполнении некоторых условий в окрестности некоторого инвариантного тора $\mathscr{I}_{1}=\mathscr{I}_{1}^{0}, \mathscr{I}_{2}=\mathscr{I}_{2}^{0}$ будет иметь структуру, отличную от полиномиальной по $\Delta I$ и $\rho$. Именно, для $N=2$

$$
H=\omega_{0} \mathscr{I}_{1}+\sum_{i=1}^{2} \mathscr{H}_{i}(\mathscr{I}, \alpha)|\rho|^{i}+O\left(\left(\left|\mathscr{I}-\mathscr{I}^{0}\right|^{3}+|\rho|^{5 / 2}\right)\right),
$$

где $|\rho|=\sqrt{\rho_{1}^{2}+\cdots+\rho_{n}^{2}}, \alpha=\left(\alpha_{1}, \ldots, \alpha_{n}\right), \alpha_{i}=\rho_{i} /|\rho|$.

Множество задач с резонансными значениями параметров относительно невелико. Тем не менее, к таким ситуациям часто приводит рассмотрение реальных многомерных квантомеханических задач. С другой стороны, если даже в исходной задаче частоты являются несоизмеримыми, но построение соответствуюших решений проводится с помощью компютера, то частоты в машинном представлении являются рациональными, т.е. всегда будут резонансными.

Заметим, что, как правило, методы построения нормальных форм опираются на тот факт, что для исходной задачи уже построены канонические переменные типа действие-угол. Существование таких переменных в окрестности изотропного тора следует 
из теоремы Дарбу-Вейнстейна (см. [13]); ясно также, что они могут быть введены различными способами. Однако вопрос о построении явных формул для них часто оказывается нетривиальным и в конкретных задачах решается по-разному. В данной работе используются явные универсальные формулы, полученные в недавней работе [9], справедливые в окрестности индивидуальньх изотропных торов.

Отметим, что в задачах с маломерными инвариантными множествами (их размерность $k<n$ (в нашем случае $k=2<n+2)$ ) существуют критические значения размерностей как инвариантного подпространства, так и системы уравнений в вариациях $(n-k)$ (в нашем случае $n+2-k$ ). Ситуация может существенно меняться при переходе от одних значений к другим. Например, для гамильтониана вида (1) при $n=1$ можно показать, что нормальная форма в окрестности некоторого инвариантного тора и в резонансном случае будет иметь вид полинома, аналогичньй (8), а при $n \geqslant 2$ - уже нет.

2. “Почти” нормальная форма четвертого порядка. Пусть при фиксированном $I_{1}^{(2)}=$ const мы выбрали некоторую функцию $F\left(\varphi_{2}^{(2)}, I_{1}^{(2)}, I_{2}^{(2)}\right)$, находящуюся в инволюции с $H_{\mathrm{osc}}$, зафиксировав тем самым выбор инвариантного двумерного тоpa. Как уже было сказано во введении, для этого тора всегда можно построить переменные типа действие-угол: $\left(\varphi^{(2)}, I^{(2)}\right) \rightarrow\left(\varphi^{(3)}, I^{(3)}\right)$. В этих переменных функция $F\left(\varphi_{2}^{(2)}, I_{1}^{(2)}, I_{2}^{(2)}\right)=F^{(3)}\left(I_{1}^{(3)}, I_{2}^{(3)}\right)$. Зафиксировав значения $I_{1}^{(3)}$ и $I_{2}^{(3)}$, мы получаем некоторый индивидуальный тор $\Lambda$. Полньй гамильтониан (1) имеет форму

$$
H^{(3)}=\omega_{0} I_{1}^{(3)}+\frac{1}{2}\left\langle r, \Gamma^{(3)}\left(\varphi^{(3)}, I^{(3)}\right) r\right\rangle
$$

где $\Gamma^{(3)}\left(\varphi^{(3)}, I^{(3)}\right)$ - гладкая $2 \pi$-периодическая функция по переменным $\left(\varphi_{1}^{(3)}, \varphi_{2}^{(3)}\right)$.

Теперь вернемся в исходное $(2 n+2)$-мерноепространство. Согласно [9] каноническая замена переменных, задаваемая с помощью формул

$$
\begin{aligned}
& \left\{\begin{array}{l}
\varphi_{1}^{(3)}=\varphi_{1}^{(4)}, \quad \varphi_{2}^{(3)}=\varphi_{2}^{(4)} \\
I_{1}^{(3)}=I_{1}^{(4)}+\Delta I_{1}^{(4)}+g_{1}, \quad I_{2}^{(3)}=I_{2}^{(4)}+\Delta I_{2}^{(4)}+g_{2}, \\
r=V\left(\varphi_{1}^{(4)}, \varphi_{2}^{(4)}, I_{1}^{(4)}, I_{2}^{(4)}\right) \xi^{(4)}+\bar{V}\left(\varphi_{1}^{(4)}, \varphi_{2}^{(4)}, I_{1}^{(4)}, I_{2}^{(4)}\right) \bar{\xi}^{(4)}
\end{array}\right. \\
& g_{m}=g_{m}\left(\varphi_{1}^{(4)}, \varphi_{2}^{(4)}, \xi^{(4)}, I_{1}^{(4)}, I_{2}^{(4)}, \bar{\xi}^{(4)}\right) \\
& \equiv \frac{1}{4 i}\left\langle V \xi^{(4)}+\bar{V} \bar{\xi}^{(4)}, \widehat{J}\left(\frac{\partial V}{\partial \varphi_{m}} \xi^{(4)}+\frac{\partial \bar{V}}{\partial \varphi_{m}} \bar{\xi}^{(4)}\right)\right\rangle, \quad m=1,2,
\end{aligned}
$$

в окрестности индивидуального тора $\Lambda$ приводит гамильтониан (11) к виду

$$
\begin{aligned}
& H=\omega_{0}\left(I_{1}^{0}+\Delta I_{1}^{(4)}\right)+\sum_{k=1}^{n} \beta_{k}\left(\varphi_{2}^{(4)}, I_{1}^{0}, I_{2}^{0}\right) \xi_{k}^{(4)} \bar{\xi}_{k}^{(4)} \\
& \quad+\frac{1}{4 i} \sum_{m=1}^{2} H_{m}^{\Gamma}\left(\Delta I_{m}^{(4)}+g_{m}\right)+O\left(\left|\xi^{(4)}\right|^{5}+\left|\Delta I^{(4)}\right|^{3}\right) \\
& H_{m}^{\Gamma}\left(\varphi^{(4)}, I^{0}, \xi^{(4)}, \bar{\xi}^{(4)}\right)=\left\langle V \xi^{(4)}+\bar{V} \bar{\xi}^{(4)}, \frac{\partial \Gamma}{\partial I_{m}^{(4)}}\left(V \xi^{(4)}+\bar{V} \bar{\xi}^{(4)}\right)\right\rangle .
\end{aligned}
$$


Итак, мы построили для гамильтониана полной системы (11) нормальную форму 2-го порядка, зависящую от угла $\varphi_{2}^{(4)}$. Теперь необходимо построить нормальную форму для членов четвертой степени по переменным $\left(\xi^{(4)}, \bar{\xi}^{(4)}\right)$ и смешанных произведений членов первого порядкапо $\Delta I_{m}^{(4)}$ и второго-по $\left(\xi^{(4)}, \bar{\xi}^{(4)}\right)$, которая тоже может зависеть от $\varphi_{2}^{(4)}$.

Лемма 1. Справедливы следующие равенства.

$$
\frac{1}{4} \int_{0}^{2 \pi}\left(\left\langle\bar{V}, \frac{\partial \Gamma}{\partial I_{m}} V\right\rangle+\left\langle V, \frac{\partial \Gamma}{\partial I_{m}} \bar{V}\right\rangle\right) d \varphi_{1}=-\frac{\partial B}{\partial I_{m}}, \quad m=1,2
$$

где матрицы $V$ и В определены формулами (6) и (7) соответственно.

$$
\frac{\partial \Gamma}{\partial I_{m}}=\left.\frac{\partial \Gamma\left(\varphi, I_{1}, I_{2}\right)}{\partial I_{m}}\right|_{I_{1}=I_{1}^{0}, I_{2}=I_{2}^{0}}
$$

ДокАЗАТЕЛьСтво. Подставим в уравнение (5) выражение (6), продифференцируем по $I_{m}$ и вьпишем по-отдельности члены при $\xi$ и $\bar{\xi}$, сгруппировав их таким образом, чтобы производные по $I_{m}$ от $V$ стояли в левой части:

$$
\begin{aligned}
& \left(\frac{d}{d t}+B-\hat{J} \Gamma\right) \frac{\partial V}{\partial I_{m}}=-\frac{\partial B}{\partial I_{m}} V+\hat{J} \frac{\partial \Gamma}{\partial I_{m}} V \\
& \left(\frac{d}{d t}-B-\hat{J} \Gamma\right) \frac{\partial \bar{V}}{\partial I_{m}}=\frac{\partial B}{\partial I_{m}} \bar{V}+\hat{J} \frac{\partial \Gamma}{\partial I_{m}} \bar{V}
\end{aligned}
$$

Умножим обе части первого уравнения на $-{ }^{t} \bar{V} \hat{J}$, второе на ${ }^{t} V \hat{J}$, и вычтем из первого второе. Последующее интегрирование полученного равенства от 0 до $2 \pi$ по переменной $\varphi_{1}$ приводит к (14).

Следующее утверждение дает нормальную форму четвертого порядка гамильтониана (13) в окрестности тора $\Lambda$.

ЛЕмма 2. Пусть при $\varphi_{2}^{(4)} \in[0,2 \pi]$ и некоторых фиксированных значениях $I_{1}^{(4)}=I_{1}^{0}, I_{2}^{(4)}=I_{2}^{0}$ выполнены условия

$$
\begin{aligned}
i \omega_{0} l+\left(\nu_{k_{1}} \beta_{k_{1}}-\nu_{k_{2}} \beta_{k_{2}}\right) \neq 0, & & |\nu|=2 \\
i \omega_{0} l+\left(\nu_{k_{1}} \beta_{k_{1}}+\nu_{k_{2}} \beta_{k_{2}}-\nu_{k_{3}} \beta_{k_{3}}-\nu_{k_{4}} \beta_{k_{4}}\right) \neq 0, & & |\nu|=4
\end{aligned}
$$

где $|\nu|=\sum_{k=1}^{n} \nu_{k}, \nu_{k} \geqslant 0, k_{1}, k_{2}, k_{3}, k_{4}=1, \ldots, n, \nu_{k}, k, l \in \mathbb{Z}$. Тогда в окрестности инвариантного двумерного тора $\Lambda$, заданного значениями $I_{1}^{0} u F$, существует 
каноническая замена переменных $\left(\varphi_{2}^{(4)}, I_{1}^{(4)}, I_{2}^{(4)}, \xi^{(4)}, \bar{\xi}^{(4)}\right) \rightarrow\left(\varphi_{2}, \mathscr{I}_{1}, \mathscr{I}_{2}, \xi, \bar{\xi}\right)$, которая приводит гамильтониан (13) к виду

$$
\begin{aligned}
H=\omega_{0} \mathscr{I}_{1} & +\sum_{k=1}^{n} \beta_{k}\left(\varphi_{2}, \mathscr{I}_{1}, \mathscr{I}_{2}\right) \xi_{k} \bar{\xi}_{k} \\
& +\sum_{k=1}^{n} \sum_{l=1}^{n} \gamma_{k l}\left(\varphi_{2}, \mathscr{I}_{1}, \mathscr{I}_{2}\right) \cdot\left(\xi_{k} \bar{\xi}_{k}\right)\left(\xi_{l} \bar{\xi}_{l}\right)+O\left(|\xi|^{5}+\left|\mathscr{I}-I^{0}\right|^{3}\right), \\
\gamma_{k l}\left(\varphi_{2}, \mathscr{I}_{1}, \mathscr{I}_{2}\right) \equiv- & \frac{1}{32 \pi} \int_{0}^{2 \pi} \sum_{m=1}^{2}\left(\sum _ { i = 2 } ^ { 3 } \sum _ { j = 2 } ^ { 3 } \left(\widehat{A}_{m k k}^{i} \widehat{G}_{m l l}^{j}+\widehat{A}_{m l l}^{i} \widehat{G}_{m k k}^{j}\right.\right. \\
& \left.+(-1)^{i+j}\left(\widehat{A}_{m k l}^{i} \widehat{G}_{m l k}^{j}+\widehat{A}_{m l k}^{i} \widehat{G}_{m k l}^{j}\right)\right) \\
& \left.+\sum_{i=1}^{4}\left(\widehat{A}_{m k l}^{i} \widehat{G}_{k l}^{5-i}+\widehat{A}_{m l k}^{i} \widehat{G}_{m l k}^{5-i}+\widehat{A}_{m k l}^{i} \widehat{G}_{m l k}^{5-i}+\widehat{A}_{m l k}^{i} \widehat{G}_{m k l}^{5-i}\right)\right) d \varphi_{1},
\end{aligned}
$$

$2 \partial e$

$$
\begin{aligned}
& \widehat{A}_{m}^{1}={ }^{t} V \frac{\partial \Gamma}{\partial I_{m}} V, \quad \widehat{A}_{m}^{2}={ }^{t} V \frac{\partial \Gamma}{\partial I_{m}} \bar{V}, \quad \widehat{A}_{m}^{3}={ }^{t} \bar{V} \frac{\partial \Gamma}{\partial I_{m}} V, \quad \widehat{A}_{m}^{4}={ }^{t} \bar{V} \frac{\partial \Gamma}{\partial I_{m}} \bar{V}, \\
& \widehat{G}_{m}^{1}={ }^{t} V \hat{J} V_{\varphi_{m}}, \quad \widehat{G}_{m}^{2}={ }^{t} V \hat{J} \bar{V}_{\varphi_{m}}, \quad \widehat{G}_{m}^{3}={ }^{t} \bar{V} \hat{J} V_{\varphi_{m}}, \quad \widehat{G}_{m}^{4}={ }^{t} \bar{V} \hat{J} \bar{V}_{\varphi_{m}}
\end{aligned}
$$

- матрицы размера $n \times n$.

Доказательство этой леммы проводится с помощью стандартной для теории нормальных форм процедуры. Для полноты изложения мы приводим его в разделе 5.

3. Усреднение по углу. Гамильтониан исходной системы приведен к форме, которая содержит угловую переменную $\varphi_{2}$. Это связано с наличием резонансного соотношения для частот. Поэтому, как было указано во введении, для устранения зависимости формы (17) от $\varphi_{2}$ построим функцию $F\left(\varphi_{2}, \mathscr{I}_{1}, \mathscr{I}_{2}\right)$, находящуюся в инволюции с гамильтонианом двумерного осциллятора $H_{\mathrm{osc}}$.

Описанная вьше процедура построения формы (17) зависит только от факта сушествования индивидуального тора и вьполнения условий (15), (16). Поэтому теперь мы можем воспользоваться полученным выражением (17) для нахождения подходящего тора $\Lambda$. Так как переменная $\varphi_{2}$ входит только в возмущение, мы возьмем в качестве функции $F$ следующее выражение:

$$
F\left(\varphi_{2}, \mathscr{I}_{1}, \mathscr{I}_{2},(\xi \bar{\xi})\right)=\sum_{k=3}^{n+2} \beta_{k} \xi_{l} \bar{\xi}_{k}+\sum_{k=3}^{n+2} \sum_{l=3}^{n+2} \gamma_{k l}\left(\xi_{k} \bar{\xi}_{k}\right)\left(\xi_{l} \bar{\xi}_{l}\right)
$$

$\beta_{l}=\beta_{l}\left(\varphi_{2}, \mathscr{I}_{1}, \mathscr{I}_{2}\right), \gamma_{k l}=\gamma_{k l}\left(\varphi_{2}, \mathscr{I}_{1}, \mathscr{I}_{2}\right)$, считая при этом, что значения $\mathscr{I}_{1}$ и произведения $\left(\xi_{k} \bar{\xi}_{k}\right)$ являются фиксированными. Теперь мы покажем, что с помощью некоторого канонического преобразования можно построить новые переменные действие-угол $(\varphi, \mathscr{I}) \rightarrow(\psi, J)$, в которых функция $F$ не будет зависеть от угловой переменной $\bmod$ $O\left((\xi+\bar{\xi})^{6}\right)$, а значит нормальная форма $(17)$ не будет зависеть от угла по крайней мере $\bmod O\left((\xi+\bar{\xi})^{5}\right)$. Сначала сделаем каноническую замену переменных $\left(\xi_{l}, \bar{\xi}_{l}\right) \rightarrow\left(\theta_{l}, \rho_{l}\right)$ : 
$\xi_{l}=\sqrt{\rho_{l} / 2} e^{i \theta_{l}}, \bar{\xi}_{l}=\sqrt{\rho_{l} / 2} e^{-i \theta_{l}}, l=1, \ldots, n$. Так как значения переменной $\mathscr{I}_{1}$ и $\rho_{l}$, $l=1, \ldots, n$, фиксированны, равенство $F=$ const задает инвариантный тор ("склееный" из замкнутых траекторий).

Функция $F$ задает одномерную гамильтонову систему в переменных $\left(\varphi_{2}, \mathscr{I}_{2}\right)$. Умножим и разделим правую часть (18) на $\rho=\sqrt{\rho_{1}^{2}+\cdots+\rho_{n}^{2}}$ и положим $\alpha=\left(\alpha_{1}, \ldots, \alpha_{n}\right)$, $\alpha_{k}=\rho_{k} / \rho, k=1, \ldots, n$. В окрестности выбранного инвариантного тора значения $\rho_{k}$ являются малыми; следовательно, мы можем считать $\rho$ малым параметром. Гамильтониан $F$ в новых обозначениях принимает вид

$$
F^{(1)} \equiv \frac{F}{\rho}=F_{0}^{(1)}\left(\varphi_{2}, I_{1}^{0}, \mathscr{I}_{2}, \alpha\right)+\rho F_{1}^{(1)}\left(\varphi_{2}, I_{1}^{0}, \mathscr{I}_{2}, \alpha\right),
$$

$F_{0}^{(1)} \equiv i \sum_{k=1}^{n} \beta_{k} \alpha_{k}, F_{1}^{(1)} \equiv \sum_{k=1}^{n} \sum_{l=1}^{n} \gamma_{k l} \alpha_{k} \alpha_{l}$. Здесь мы снова используем верхний индекс в скобках для обозначения номера замены переменных. Для приведения гамильтониана (19) к нормальной форме можно воспользоваться обычными методами усреднения [1], [2], [4], [5]. Для этого введем новые канонические переменные действие-угол традиционным способом:

$$
\begin{aligned}
J_{2} & =\left.\frac{1}{2 \pi} \int_{0}^{2 \pi} \mathscr{I}_{2} d \varphi_{2}\right|_{F_{0}^{(1)}\left(\varphi_{2}, I_{1}^{0}, \mathscr{I}_{2}\right)}=\text { const } \\
\psi_{2} & =\left.\frac{\partial}{\partial J_{2}} \int_{0}^{\varphi} \mathscr{I}_{2}\left(J_{2}\right) d \tau\right|_{F_{0}^{(1)}\left(\varphi_{2}, I_{1}^{0}, \mathscr{I}_{2}\left(J_{2}\right)\right)}=\text { const } .
\end{aligned}
$$

Тогда гамильтониан (19) принимает вид

$$
F^{(2)}=F_{0}^{(2)}\left(I_{1}^{0}, J_{2}, \Delta I_{2}^{(2)}, \alpha\right)+\rho F_{1}^{(2)}\left(\psi_{2}, I_{1}^{0}, J_{2}, \alpha\right) .
$$

ЗАмЕчАниЕ. В общем случае зависимость $F_{0}^{(2)}$ от параметров $\alpha_{k}$ носит нерегулярный характер. В противном случае, каноническое преобразование, задаваемое формулами (20) уничтожало бы зависимость от $\psi_{2}$ во всех $\beta_{l}, l=1, \ldots, n$, одновременно при переходе от угла $\varphi_{2} \mathrm{k} \psi_{2}$.

Так как $\beta_{k} \neq 0, k=1, \ldots, n$, а $\rho_{k}$ не равны нулю одновременно, то $\partial F_{0}^{(2)} / \partial J_{2} \neq 0$. Тогда существует каноническое преобразование $\left(\psi_{2}, J_{2}\right) \rightarrow\left(\psi_{2}^{(1)}, J_{2}^{(1)}\right)$, приводящее $F^{(2)}$ к виду

$$
F^{(3)}=F_{0}^{(3)}\left(I_{1}^{0}, J_{2}^{(1)}, \alpha\right)+\rho F_{1}^{(3)}\left(I_{1}^{0}, J_{2}^{(1)}, \alpha\right)+O\left(\rho^{2}\right),
$$

где $F_{1}^{(3)}=(1 /(2 \pi)) \int_{0}^{2 \pi} F_{1}^{(2)}\left(\psi_{2}, I_{1}^{0}, J_{2}^{(1)}, \alpha\right) d \psi_{2}$.

Таким образом, мы получили комбинацию канонических преобразований, которая уничтожает зависимость от угла $\varphi_{2}$ в $(19)$ до членов порядка $O\left(\rho^{2}\right)$. Переобозначим $\psi_{2}^{(1)}=\varphi_{2}, J_{2}^{(1)}=I_{2}$.

Теорема 1. В окрестности двумерного тора в четырехмерном фазовом пространстве $\mathbb{R}_{\varphi, I^{0}}^{4}$, заданного фиксированным значением $I_{1}^{(2)}=I_{1}^{0}$ (см. (9)) и условием $F\left(\varphi_{2}, I_{1}^{0}, I_{2}, \rho^{0}\right)=$ const, где $F$ задана формулой (18) при некоторых фиксированных значениях $\rho_{l}^{0}, l=1, \ldots, n$, гамильтониан (1) приводится $\kappa$ виду (10) при условии, что для всех точек этого тора выполнены условия нерезонанснос$m u(15),(16)$. 
4. Четырехмерный ангармонический осциллятор. В формуле (17) мы ограничиваемся четвертьм порядком по переменным $(\xi, \bar{\xi})$. Поэтому набор резонансных соотношений конечен. В силу непрерывности функций $\beta_{k}, k=1, \ldots, n$, соответствующие множества резонансных точек $\left(\psi_{2}^{(1)}, J_{2}^{(1)}\right)$ могут образовьвать либо замкнутые, либо не замкнутые $2 \pi$-периодические по $\psi_{2}^{(1)}$ кривые. Соответственно, множество нерезонансных точек лежит между этими кривыми и будет открытым. Указанная процедура "склейки" тора и последующего усреднения по нему возможна только в том случае, если линии уровня в формулах (20) не пересекаются и не касаются с резонансными кривыми. Несмотря на конечность набора резонансных соотношений, это требование может существенно уменьшить число торов, для которых возможно построение нормальной формы (10). Более того, может так случиться, что условия (15), (16) будут справедливы лишш для интегрируемых случаев (например, для гармонического осциллятора). Пример, рассмотренньй ниже, показывает, что условия $(15),(16)$ могут быть вьполнены, а нормальная форма (10) может быть построена для нетривиальных ситуаций.

Рассмотрим четырехмерный ангармонический осциллятор с гамильтонианом, задаваемьм формулой (11), где

$$
\Gamma(\varphi, I)=\left(\begin{array}{cccc}
\omega_{3}^{2}\left(1+2 \varepsilon f_{3}(\varphi, I) / \omega_{3}\right) & 0 & 0 & 0 \\
0 & \omega_{4}^{2}\left(1+2 \varepsilon f_{4}(\varphi, I) / \omega_{4}\right) & 0 & 0 \\
0 & 0 & 1 & 0 \\
0 & 0 & 0 & 1
\end{array}\right)
$$

$\varepsilon$ предполагается малой фиксированной константой, $f_{3}, f_{4}$ - аналитические $2 \pi$-периодические по углам функции. Например, в рассматриваемом ниже примере функции $f_{3}$ и $f_{4}$ получены из частот $\Omega_{i}^{2}\left(Q_{1}, Q_{2}\right)=\omega_{i}\left(Q_{1}^{2}+Q_{2}^{2}\right) / 2, i=3,4$, при помощи преобразований $(3),(9)$.

Согласно теории $\Phi$ локе-Ляпунова [10] матрица показателей быть представлена в виде

$$
B\left(\varphi_{2}, I_{1}, I_{2}\right)=\left(\begin{array}{cc}
\omega_{3}+\varepsilon\left\langle f_{3}\right\rangle+O\left(\varepsilon^{2}\right) & 0 \\
0 & \omega_{4}+\varepsilon\left\langle f_{4}\right\rangle+O\left(\varepsilon^{2}\right)
\end{array}\right),
$$

где

$$
\left\langle f_{i}\right\rangle \equiv\left\langle f_{i}\left(\varphi_{2}, I_{1}, I_{2}\right)\right\rangle=\frac{1}{2 \pi} \int_{0}^{2 \pi} f_{i}\left(\varphi_{1}, \varphi_{2}, I_{1}, I_{2}\right) d \varphi_{1}, \quad i=3,4
$$

Функции $\beta_{3}$ и $\beta_{4}$ при $\varepsilon>0$ являются функциями $\left(\varphi_{2}, I_{1}, I_{2}\right), 2 \pi$-периодическими по $\varphi_{2}$. Переменная $I_{1}$ фиксирована значением энергии системы, поэтому произвол остался в выборе $\left(\varphi_{2}, I_{2}\right)$.

На первом этапе не будем принимать в рассмотрение слагаемые порядка $O\left(\varepsilon^{2}\right)$. При $\varepsilon=0$ показатели $\Phi$ локе $\beta_{3}$ и $\beta_{4}$ равны частотам $\omega_{3}$ и $\omega_{4}$ соответственно. Тогда множества резонансных значений $\left(\beta_{3}, \beta_{4}\right)$ образуют прямые на плоскости $\left(\omega_{3}, \omega_{4}\right)$. В силу замкнутости области определения $\beta_{3}$ и $\beta_{4}$ значения $\beta_{3}$ и $\beta_{4}$ ограничены сверху и снизу. Согласно формуле (24) эти ограничения определяются минимумами и максимумами функций $\left\langle f_{3}\right\rangle$ и $\left\langle f_{4}\right\rangle$. Введем следующее обозначение: $\left\langle\hat{f}_{i}\right\rangle=\max _{\left(\varphi_{2}, I_{2}\right)}\left|\left\langle f_{i}\right\rangle\right|$. Справедливы следующие оценки: $\beta_{3}-\omega_{3}^{0} \in\left[-\varepsilon\left\langle\hat{f}_{3}\right\rangle, \varepsilon\left\langle\hat{f}_{3}\right\rangle\right]$ и $\beta_{4}-\omega_{4}^{0} \in\left[-\varepsilon\left\langle\hat{f}_{4}\right\rangle, \varepsilon\left\langle\hat{f}_{4}\right\rangle\right]$. В 
процессе построения нормальной формы, не зависящей от угла $\varphi_{2}$, значения показателей $\Phi$ локе останутся в этих интервалах. Пусть $R$ - радиус окрестности нерезонансной точки $\left(\omega_{3}^{0}, \omega_{4}^{0}\right)$, точки которой также являются нерезонансными. Тогда при

$$
\varepsilon<\varepsilon_{0}, \quad \varepsilon_{0}=\frac{R}{\sqrt{\left\langle\hat{f}_{3}\right\rangle^{2}+\left\langle\hat{f}_{4}\right\rangle^{2}}}
$$

значения $\left(\beta_{3}, \beta_{4}\right)$ принадлежат нерезонансной окрестности точки $\left(\omega_{3}^{0}, \omega_{4}^{0}\right)$.

Теперь примем во внимание слагаемые порядка $O\left(\varepsilon^{2}\right)$. В соответствии со стандартной теорией возмущений в силу непрерывности всех функций следует, что если соотношения вьполнены при некотором $\varepsilon_{0}$, то наличие поправок следуюших порядков по $\varepsilon_{0}$ приведет к сужению окрестности на величину порядка $O\left(\varepsilon_{0}^{2}\right)$. Таким образом, мы показали существование некоторой нерезонансной окрестности точки $\left(\omega_{3}^{0}, \omega_{4}^{0}\right)$, а значит и торов, порождающих форму (17), не зависящую от угла $\varphi_{2}$.

Проиллюстрируем сказанное примером численного построения соответствующей нормальной формы для четырехмерного ангармонического осциллятора (23) с функциями $f_{i}=2 \omega_{i}\left(n_{1} I_{1}+m_{2} I_{2}\right) / \omega_{1}+2 \omega_{i}\left(n_{2} I_{1}-m_{1} I_{2}\right) / \omega_{2}, i=3,4$. На рис. 1 представлены линии уровня функции $F$, определенной в (18), а также резонансные кривые.

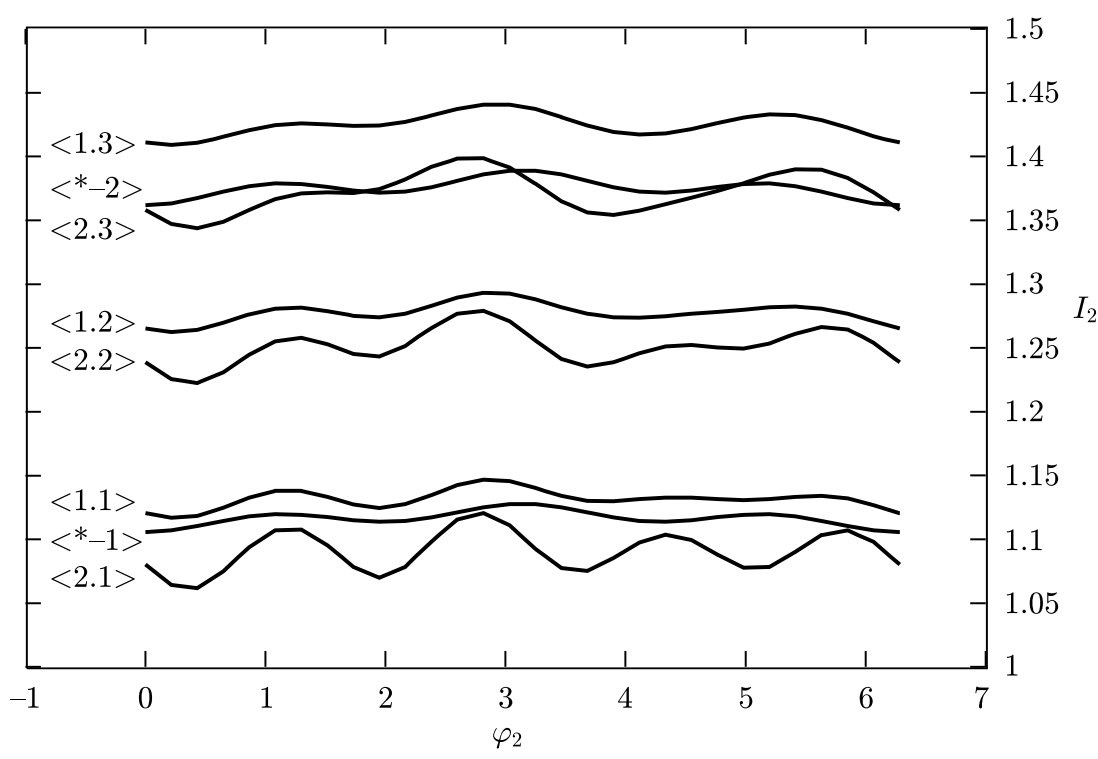

Рис. 1. Линии уровня и резонансные кривые

Номерами $\langle 1.1\rangle-\langle 1.3\rangle$ обозначены кривые соответствуюшие фиксированньп значениям $\rho=(1.5,0.5)$, а $\langle 2.1\rangle-\langle 2.3\rangle-$ значениям $\rho=(0.5,1.5)$. Номерами $\langle *-1\rangle-\langle *-2\rangle$ обозначены резонансные кривые. Как видно из рис. 1 , имеются пересечения с резонансной кривой только для кривой $\langle 2.3\rangle$. Это означает, что для этой кривой описанная процедура "склейки" тора не справедлива.

На рис. 2 представлены проекции двумерных инвариантных торов в четырехмерном фазовом пространстве гармонического осциллятора, “склеенных" с помощью кривых $\langle 1.2\rangle$ и $\langle 2.2\rangle$, на конфигурационное пространство двумерного гармонического осциллятора. Видно, что проекции торов, соответствующих “близким" значениям параметра $I_{2}$, 


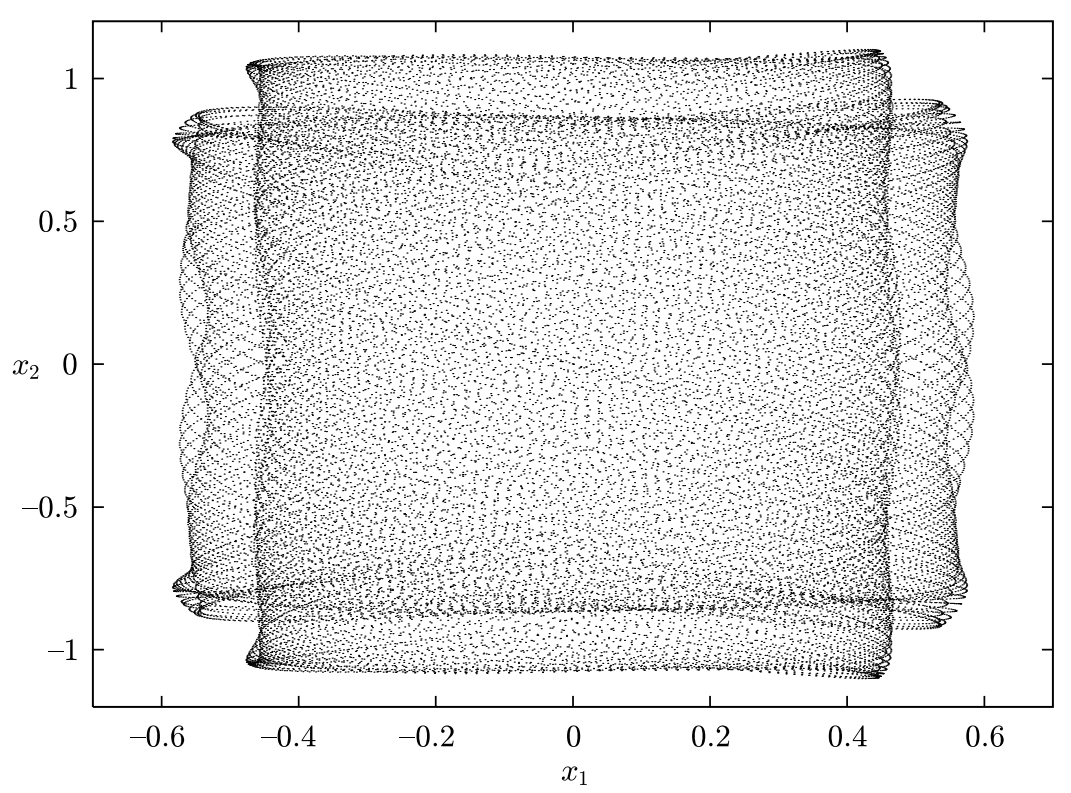

Рис. 2. Проекции инвариантного тора

имеют “искривленные” края, форма которых довольно существенно различается. В нерезонансном случае такие проекции “одинаковые" прямоугольники, всюду плотно покрываемые незамкнутыми траекториями.

В заключение этого раздела отметим, что при построении нормальных форм более высокого порядка число резонансных соотношений увеличивается и, соответственно, множество инвариантных торов, построенных с помощью предложенной процедуры, может уменьшаться. Более того, при $N \rightarrow \infty$ это множество вообще может исчезнуть. Однако с точки зрения приложения к спектральньм задачам квантовой механики интерес представляет возможность построения инвариантных торов и нормальных форм для конечных $N$. Полезные следствия возникают уже при $N=4$. Собственно, рассматриваемые здесь вопросы возникли при построении асимптотических собственных значений и функций операторов Шрёдингера (см. также [12], [14], [16], [17]).

\section{5. Вычисление коэффициентов нормальной формы четвертого порядка.}

ДоКАЗАТЕЛЬСТво ЛЕМмы 2. Коэффициенты $\gamma_{k l}$ получаются в результате стандартной процедуры нормализации [4], [5], основанной на использовании производящей функции и равенства (14). Для того, чтобы привести гамильтониан (13) к виду (17), воспользуемся производящей функцией, что автоматически обеспечит каноничность преобразования, и выведем необходимые и достаточные условия существования этого преобразования.

Рассмотрим производящую функцию $S$ в следующем виде:

$$
\begin{aligned}
S & =\varphi^{(4)} \Delta I^{(5)}+\xi^{(4)} \bar{\xi}^{(5)}+S_{2}+S_{4} \\
S_{2} & =\sum_{m=1}^{2} \sum_{l=-\infty}^{\infty} \sum_{k_{1}=1}^{n} \sum_{k_{2}=1}^{n} \sum_{|\nu|=2} S_{m l k_{1} k_{2} \nu_{k_{1}} \nu_{k_{2}}}^{2} \cdot \Delta I_{m}^{(5)}\left(\xi_{k_{1}}^{(4)}\right)^{\nu_{k_{1}}\left(\bar{\xi}_{k_{2}}^{(5)}\right)^{\nu_{k_{2}}} e^{i l \varphi_{1}^{(4)}}}
\end{aligned}
$$




$$
\begin{aligned}
& S_{4}=\sum_{l=-\infty}^{\infty} \sum_{k_{1}=1}^{n} \sum_{k_{2}=1}^{n} \sum_{k_{3}=1}^{n} \sum_{k_{4}=1}^{n} \sum_{|\nu|=4} S_{l k_{1} k_{2} k_{3} k_{4} \nu_{k_{1}} \nu_{k_{2}} \nu_{k_{3}} \nu_{k_{4}}}
\end{aligned}
$$

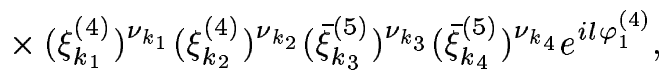

где

$$
\begin{aligned}
& |\nu|=\sum_{k=1}^{n} \nu_{k}, \quad \nu_{k} \geqslant 0, \quad S_{m l k_{1} k_{2} \nu_{k_{1}} \nu_{k_{2}}}^{2}=S_{m l k_{1} k_{2} \nu_{k_{1}} \nu_{k_{2}}}^{2}\left(\varphi_{2}^{(4)}, I^{0}\right),
\end{aligned}
$$

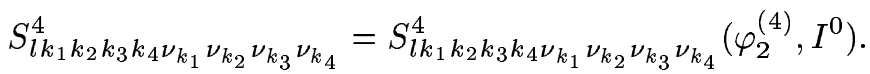

Тогда переменные $\left(\varphi^{(5)}, \Delta I^{(4)}, \xi^{(5)}, \bar{\xi}^{(4)}\right)$ выражаются следующим образом:

$$
\left\{\begin{array}{l}
\varphi^{(5)}=\varphi^{(4)} \\
\Delta I_{m}^{(4)}=\Delta I_{m}^{(5)}+\frac{\partial S_{2}}{\partial \varphi_{m}^{(4)}}+\frac{\partial S_{4}}{\partial \varphi_{m}^{(4)}}+O\left(\left(\xi^{(4)}+\bar{\xi}^{(5)}\right)^{4}+\left(\Delta I^{(5)}\right)^{5}\right), m=1,2 \\
\bar{\xi}^{(4)}=\bar{\xi}^{(5)}+\frac{\partial S_{2}}{\partial \xi^{(4)}}+\frac{\partial S_{4}}{\partial \xi^{(4)}}+O\left(\left(\xi^{(4)}+\bar{\xi}^{(5)}\right)^{4}+\left(\Delta I^{(5)}\right)^{5}\right) \\
\xi^{(5)}=\xi^{(4)}+\frac{\partial S_{2}}{\partial \bar{\xi}^{(5)}}+\frac{\partial S_{4}}{\partial \bar{\xi}^{(5)}}+O\left(\left(\xi^{(4)}+\bar{\xi}^{(5)}\right)^{4}+\left(\Delta I^{(5)}\right)^{5}\right)
\end{array}\right.
$$

После подстановки в гамильтонианы (13), (17), мы получаем следующие уравнения:

$$
\begin{aligned}
& \omega_{0} \frac{\partial S_{2}}{\partial \varphi_{1}^{(4)}}+\left(\left\langle B \xi^{(4)}, \frac{\partial}{\partial \xi^{(4)}}\right\rangle-\left\langle B \bar{\xi}^{(5)}, \frac{\partial}{\partial \bar{\xi}^{(5)}}\right\rangle\right) S_{2} \\
& =\sum_{m=1}^{2}\left(\left\langle\xi^{(4)}, \frac{\partial B}{\partial I_{m}^{(5)}} \bar{\xi}^{(5)}\right\rangle-H_{m}^{\Gamma}\left(\varphi^{4}, I^{0}, \xi^{(4)}, \bar{\xi}^{(5)}\right)\right) \Delta I_{m}^{(5)} \\
& \omega_{0} \frac{\partial S_{4}}{\partial \varphi_{1}^{(4)}}+\left(\left\langle B \xi^{(4)}, \frac{\partial}{\partial \xi^{(4)}}\right\rangle-\left\langle B \bar{\xi}^{(5)}, \frac{\partial}{\partial \bar{\xi}^{(5)}}\right\rangle\right) S_{4} \\
& =\sum_{k=1}^{n} \sum_{l=1}^{n} \gamma_{k l}\left(\xi_{k}^{(4)} \bar{\xi}_{k}^{(5)}\right)\left(\xi_{l}^{(4)} \bar{\xi}_{l}^{(5)}\right)-\frac{1}{4 i} \sum_{m=1}^{2} H_{m}^{\Gamma}\left(\varphi^{4}, I^{0}, \xi^{(4)}, \bar{\xi}^{(5)}\right) g_{m}
\end{aligned}
$$

Используя представления (25) и (26), мы приравниваем слагаемые одинаковой степени по переменньм $\Delta I_{1}^{(5)}, \Delta I_{2}^{(5)}, \xi^{(4)}, \bar{\xi}^{(5)}$. Предположим, что вьполнены условия разрешимости для уравнений (28), (29). Так как по условию на нашем индивидуальном торе выполнены условия (15), (16), используя формулу (14) из леммы 1 , мы решаем систему (28), (29) и находим производящую функцию $S$. Подставляя ее в $(27)$ и разрешая полученную систему относительно переменных $\varphi^{(5)}, \Delta I^{(5)}, \xi^{(5)}, \bar{\xi}^{(5)}$, получаем каноническое преобразование $\left(\varphi^{(4)}, \Delta I^{(4)}, \xi^{(4)}, \bar{\xi}^{(4)}\right) \rightarrow\left(\varphi^{(5)}, \Delta I^{(5)}, \xi^{(5)}, \bar{\xi}^{(5)}\right)$, приводящее гамильтониан (13) к виду

$$
\begin{aligned}
H= & \omega_{0}\left(I_{1}^{0}+\Delta I_{1}^{(5)}\right)+\sum_{k=1}^{n} \beta_{k} \xi_{k}^{(5)} \bar{\xi}_{k}^{(5)}+\sum_{k=1}^{n}\left(\sum_{m=1}^{2} \mu_{m k} \Delta I_{m} \xi_{k}^{(5)} \bar{\xi}_{k}^{(5)}\right. \\
& \left.+\sum_{l=1}^{n} \gamma_{k l} \cdot\left(\xi_{k}^{(5)} \bar{\xi}_{k}^{(5)}\right)\left(\xi_{l}^{(5)} \bar{\xi}_{l}^{(5)}\right)\right)+O\left(\left|\xi^{(5)}\right|^{5}+\left|\Delta I^{(5)}\right|^{3}\right)
\end{aligned}
$$


где $\beta_{k}=\beta_{k}\left(\varphi_{2}^{(5)}, I_{1}^{(5)}, I_{2}^{(5)}\right), \mu_{m k}=\mu_{m k}\left(\varphi_{2}^{(5)}, I_{1}^{0}, I_{2}^{0}\right) \equiv \partial \beta_{k}\left(\varphi_{2}^{(5)}, I_{1}^{0}, I_{2}^{0}\right) / \partial I_{m}, \gamma_{k l}=$ $\gamma_{k l}\left(\varphi_{2}^{(5)}, I_{1}^{(5)}, I_{2}^{(5)}\right)$ определена в условиях леммы.

Заметим, что все произведенные замены переменных были каноническими в некоторой окрестности индивидуального тора, заданного $\left(I_{1}^{0}, I_{2}^{0}\right)$. Полученная в результате форма гамильтониана (30) с точностью до членов порядка $O\left(\left|\xi^{(5)}\right|^{5}+\left|\Delta I^{(5)}\right|^{3}\right)$ описывает исходньй гамильтониан в указанной окрестности. Введем теперь переменные $\mathscr{I}_{1}$ и $\mathscr{I}_{2}$ следующим образом: $\mathscr{I}_{1}=I_{1}^{0}+\Delta I_{1}^{(5)}, \mathscr{I}_{2}=I_{2}^{0}+\Delta I_{2}^{(5)}$. Подставим в $(30)$ и воспользуемся формулой $f\left(x_{0}\right)+\partial f / \partial x \cdot \Delta x+O\left(|\Delta x|^{3}\right)=f\left(x_{0}+\Delta x\right)+O\left(|\Delta x|^{3}\right)$ при $\partial^{2} f / \partial x^{2}=0$. Если теперь опустить индексы шага приведения у переменных, композиция последовательных канонических преобразований $(12),(27)$ при выполнении условий (15), (16) обеспечивает приведение гамильтониана (13) к виду (17). Лемма доказана.

\section{СПИСОК ЦИТИРОВАННОЙ ЛИТЕРАТУРЫ}

[1] Арнольд В.И. Малые знаменатели и проблемы устойчивости в классической и небесной механике // УМН. 1963. Т. 18. №6. С. 91-192.

[2] Арнольд В. И. Математические методы классической механики. М.: Наука, 1974.

[3] Broer H. W., Huitema G. B., Sevryuk M. B. Quasi-Periodic Motions in Families of Dynamical Systems // Lecture Note in Math. V. 1645. Berlin: Springer, 1996.

[4] Брюно А. Д. Ограниченная задача трех тел: плоские периодические орбиты. М.: Наука, 1990.

[5] Джакалья Г. Е. О. Методы теории возмущений для нелинейных систем. М.: Наука, 1979.

[6] Jorba A., Llave R. de la, Zou M. Linstedt series for lower dimensional tori of hamiltonian systems with three or more degrees of freedom // NATO Adv. Sci. Inst. Ser. C. Math. Phys. Sci. (S Agaro, Spain, June 1995) / ed. C. Simo, 1995.

[7] Rüssmann H. Invariant tori in non-degenerate nearly integrable Hamiltonian systems // Regular and Chaotic Dynamics. 2001. V. 6. № 2. P. 119-204.

[8] Белов В. В., Доброхотов О.С., Доброхотов С. Ю. Изотропные торы, комплексный росток и индекс Маслова, нормальные формы и квазимоды в многомерных спектральных задачах // Матем. заметки. 2001. Т. 69. № 4. С. 483-514.

[9] Белов В.В., Доброхотов С. Ю., Максимов В. А. Явные формулы для обобщенных переменных действие-угол в окрестности изотропного тора и их применение // ТМФ. 2003. T. 135. № 3. С. 378-408.

[10] Якубович В. А., Старжинский В. М. Линейные дифференциальные уравнения с периодическими коэффициентами. М.: Наука, 1972.

[11] Боголюбов Н.Н., Митропольский Ю. А. Асимптотические методы в теории колебаний. М.: Наука, 1974.

[12] Карасев М. В. Квантовая редукция на орбиты алгебр // Препринт ИТФ-87-157Р. Киев: Ин-т теор. физ. АН УССР, 1988.

[13] Арнольд В. И., Гивенталь А. Б. Симплектическая геометрия. Ижевск: РХД, 2000.

[14] Dobrokhotov S. Yu., Shafarevich A. I Quantum selection of isotropic partially integrable Hamiltonian systems in quasiclassical approximation // Russian J. Math. Phys. 1998. V. 5. № 2. Р. 267-272.

[15] Доброхотов С. Ю., Оливэ В. М. Замкнутые траектории и двумерные торы в квантовой проблеме для трехмерного ангармонического осциллятора // Тр. МMO. 1997. Т. 51. С. 3-87.

[16] Dobrokhotov S. Yu., Olive Martinez V., Shafarevich A. I Closed trajectories and two-dimensional tori in the quantum problem for a three-dimensional resonant anharmonic oscillator // Russian J. Math. Phys. 1995. V. 3. № 1. P. 133-138.

[17] Карасев М. В. Новые глобальные асимптотики и аномалии в задачах квантования адиабатических инвариантов // Функцион. анализ и его прилож. 1990. Т. 24. № 2. С. 24-36. 